\title{
Measures of Complexity
}

\section{a non--exhaustive list}

\section{Seth Lloyd}

\author{
d'Arbeloff Laboratory for Information Systems and Technology \\ Department of Mechanical Engineering \\ Massachusetts Institute of Technology \\ slloyd@mit.edu
}

The world has grown more complex recently, and the number of ways of measuring complexity has grown even faster. This multiplication of measures has been taken by some to indicate confusion in the field of complex systems. In fact, the many measures of complexity represent variations on a few underlying themes. Here is an (incomplete) list of measures of complexity grouped into the corresponding themes.

An historical analog to the problem of measuring complexity is the problem of describing electromagnetism before Maxwell's equations. In the case of electromagnetism, quantities such as electric and magnetic forces that arose in different experimental contexts were originally regarded as fundamentally different. Eventually it became clear that electricity and magnetism were in fact closely related aspects of the same fundamental quantity, the electromagnetic field. Similarly, contemporary researchers in architecture, biology, computer science, dynamical systems, engineering, finance, game theory, etc., have defined different measures of complexity for each field. Because these researchers were asking the same questions about the complexity of their different subjects of research, however, the answers that they came up with for how to measure complexity bear a considerable similarity to eachother. Three questions that researchers frequently ask to quantify the complexity of the thing (house, bacterium, problem, process, investment scheme) under study are

1. How hard is it to describe?

2. How hard is it to create?

3. What is its degree of organization?

Here is a list of some measures of complexity grouped according to the question that they try to answer. Measures within a group are typically closely related quantities.

1. Difficulty of description. Typically measured in bits.

Information;

Entropy;

Algorithmic Complexity or Algorithmic Information Content;

Minimum Description Length;

Fisher Information; 


\section{Renyi Entropy;}

Code Length (prefix-free, Huffman, Shannon-Fano, error-correcting, Hamming);

Chernoff Information;

Dimension;

Fractal Dimension;

Lempel--Ziv Complexity.

2. Difficulty of creation. Typically measured in time, energy, dollars, etc.

Computational Complexity;

Time Computational Complexity;

Space Computational Complexity;

Information--Based Complexity;

Logical Depth;

Thermodynamic Depth;

Cost;

Crypticity.

3. Degree of organization. This may be divided up into two quantities: a) Difficulty of describing organizational structure, whether corporate, chemical, cellular, etc.; b) Amount of information shared between the parts of a system as the result of this organizational structure.

a) Effective Complexity

Metric Entropy; Fractal Dimension; Excess Entropy;

Stochastic Complexity;

Sophistication;

Effective Measure Complexity;

True Measure Complexity;

Topological epsilon-machine size;

Conditional Information;

Conditional Algorithmic Information Content;

Schema length;

Ideal Complexity;

Hierarchical Complexity;

Tree subgraph diversity; 


\section{Homogeneous Complexity;}

Grammatical Complexity.

b) Mutual Information:

Algorithmic Mutual Information;

Channel Capacity;

Correlation;

Stored Information;

Organization.

In addition to the above measures, there are a number of related concepts that are not quantitative measures of complexity per se, but that are closely related. Such concepts include

Long--Range Order;

Self--Organization;

Complex Adaptive Systems;

Edge of Chaos.

Please feel free to send me additions to this list, whether or not they fall in the classification scheme.

Seth Lloyd, slloyd@mit.edu 